\title{
On the Range of Feasible Power Trajectories for a Population of Thermostatically Controlled Loads
}

\author{
Dario Paccagnan, Maryam Kamgarpour and John Lygeros
}

\begin{abstract}
We study the potential of a population of thermostatically controlled loads to track desired power signals with provable guarantees. Based on connecting the temperature state of an individual device with its internal energy, we derive necessary conditions that a given power signal needs to satisfy in order for the aggregation of devices to track it using non-disruptive probabilistic switching control. Our derivation takes into account hybrid individual dynamics, an accurate continuous-time Markov chain model for the population dynamics and bounds on switching rates of individual devices. We illustrate the approach with case studies.
\end{abstract}

\section{INTRODUCTION}

Increasing penetration of renewable energy and liberalization of electricity markets are significantly changing power system operations. Since in a power grid demand and supply must at all times be balanced, the unpredictability in the renewable energy generation increases the need for ancillary services, which are today mainly covered by conventional generators. With the advancement of smart grid sensing and communication infrastructure, household appliances can provide new means for ancillary services. For example, the power consumption of a population of Thermostatically Controlled Loads (TCLs) can follow a desired trajectory obtained based on the fluctuation of renewable energy generation [1].

A main challenge in the participation of a population of TCLs in ancillary service markets is characterizing the set of power trajectories that can be tracked with provable guarantees. Once this set is determined, control schemes need to be developed so that the population can track any power signal within this set with a desired accuracy. The objective of this paper is to find fundamental limits on the set of power trajectories that can be tracked by an aggregation of TCLs. To do so, a dynamical model for power consumption of the population of TCLs needs to be developed. While an individual TCL can be described as a hybrid dynamical system, developing an accurate population description that is simple enough for analysis and control design is challenging.

Previous work has extensively studied developing population dynamics for TCLs. In [2] the probability density function (PDF) of a homogenous aggregation of TCLs is characterized. In [1] solutions to the PDF were derived and a linear model was developed to account for heterogeneity and control of the population by varying their temperature set points. Discrete-time Markov chain abstractions of the PDF

This work was supported by Swiss Nano-Tera project HeatReserves and Swiss Competence Centers for Energy Research FEEB\&D.

D. Paccagnan, M. Kamgarpour and J. Lygeros are with the Automatic Control Laboratory, ETH Zürich, Switzerland. Email: \{dariop, mkamgar, lygeros\}@control.ee.ethz.ch. were developed in [3], [4]. A more detailed description based on a two-dimensional hybrid system is proposed in [5] and the corresponding PDF evolution is studied. All the above approaches use the aggregate dynamics to design controllers to track a given power trajectory, but do not a priori quantify the set of power signals that the population can track.

The mean field approach has been applied to a population of TCLs in order to develop decentralized control schemes [6], [7]. Here, each TCL optimizes a cost function that reflects its desired behavior, such as minimal consumption, and a term accounting for its contribution to the aggregate power consumption. Linear dynamic is assumed for individual TCLs and the switching between the on/off modes is removed. A similar framework is also developed in [8], where the authors address the problem of ensuring that the power grid frequency is maintained within required bounds.

Recently, a storage model for a population of TCLs was introduced in [9], [10]. Here, the hybrid dynamics of TCLs is approximated, and then a generalized battery model for an aggregation of these was introduced. The authors provide necessary and sufficient conditions to characterize the set of feasible power trajectories for the simplified dynamics. The performance was validated on a realistic simulation with hybrid dynamics. Our work is inspired by this approach.

The main objective of this paper is to characterize the set of feasible power trajectories of a population of TCLs, using accurate individual models of the TCLs. As such, this paper has three contributions. First, we derive a continuous-time Markov chain description of a population of TCLs under probabilistic switching control. We use this to characterize the limits of performance of population control of TCLs by analyzing the transients and steady-state distributions of the controlled dynamics. Second, by connecting the energy state of individual TCLs with the average temperature of the aggregation, we characterize the set of feasible trajectories, in horizon of minutes to hours, under rate limited switching control. Third, to illustrate the approach, we develop a simple controller to track a given power trajectory. The derived bounds on feasible trajectories are useful for ancillary services such as load following or energy arbitrage [10].

The paper is organized as follows. In Section II we detail the individual and population model. In Section III we discuss the discretization scheme and analyze the transients and steady states of the controlled population. In Section IV we develop bounds on the feasible power trajectories under rate-limited probabilistic switching control. In Section $\mathrm{V}$, we study the effectiveness of our bounds using numerics. In Section VI we conclude and summarize future work. 


\section{IndividuAl AND AgGREgATE Models FOR TCLs}

\section{A. Individual TCL Model}

Thermostatically controlled loads are household appliances, such as electric heaters, water heaters, and air conditioners, that operate in two discrete modes: on and off. When in the on mode, the device consumes a constant amount of power to cool down or to heat up the space. In the off mode no electricity is used and the temperature naturally evolves towards the ambient temperature. Given the discrete dynamics of the mode and the continuous evolution of the temperature, an individual TCL is modeled as a hybrid system. The state of a single unit is characterized by the pair $(X, m) \in \mathbb{R} \times\{0,1\}$ representing the temperature and the mode. The continuous dynamics is governed by the following stochastic differential equation

$$
d X=\left(-\frac{1}{C R}\left(X-\theta_{a}\right) \pm m \frac{\eta P}{C}\right) d t+\sigma d \omega,
$$

where the signs + and - refer respectively to heating and cooling devices. The first term in the drift from (1) takes into account the ambient losses, $\frac{\eta P}{C}$ represents the input from the TCL, $\sigma>0$ and $d \omega$ is a Wiener process capturing model uncertainties. The parameters $C, R, \theta_{a}, P, \eta \in \mathbb{R}_{+}$are thermal capacitance and resistance, ambient temperature, electric power, and coefficient of performance, respectively. In the following, let us consider a cooling unit and use subscripts 1 and 0 to refer to the on and off modes respectively.

Each TCL is designed to operate between a temperature deadband $\left[\theta_{-}, \theta_{+}\right]$and the discrete state transition is usually modelled with deterministic hard boundaries of the form

$$
m(t+d t)= \begin{cases}1, & X(t) \geq \theta_{+} \\ m(t), & X(t) \in\left(\theta_{-}, \theta_{+}\right) \\ 0, & X(t) \leq \theta_{-}\end{cases}
$$

see for example [2] and [1]. In this work we use soft boundaries and define the transition probability from one mode to the other as

$\mathbb{P}(m(t+d t)=i \mid m(t)=j, X(t)=x)=\lambda_{j i}(x) d t+\mathcal{O}(d t)$,

with $i \neq j, i, j=0,1$. In particular, the rates $\lambda_{10}(x), \lambda_{01}(x)$ : $\mathbb{R} \rightarrow \mathbb{R}_{+}$are taken to be zero respectively in the intervals $\left[\theta_{-},+\infty\right)$ and $\left(-\infty, \theta_{+}\right]$, while they grow quickly outside these intervals as Figure 1 exemplifies.

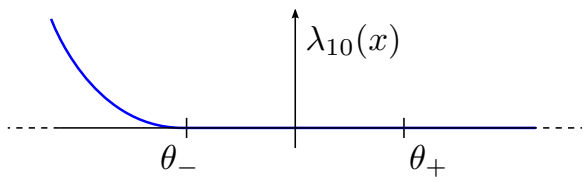

on

Fig. 1: Example of rate $\lambda_{10}(x)$ for the soft boundaries.

The choice of soft boundaries has been made for two reasons. First, each thermostat does not instantaneously detect when the boundary of the deadband is reached, introducing some delay before the switch is triggered. Second, the resulting model qualitatively takes into account heterogeneity in the deadband interval, i.e. the fact that different units may have slightly different deadbands. Furthermore, it has been shown that the approximated stochastic process using soft boundaries converges in distribution to that using hard boundaries as the switching rates near the boundaries increase [11]. For the problem at hand, the stationary distribution corresponding to hard and soft boundaries are compared in Figure 3.

Throughout the paper, we use the values of the physical constants as in the following table, representing typical residential air conditioning (AC) systems [10].

TABLE I: Typical parameter values for residential ACs.

\begin{tabular}{c|c|c|c|c|c|c|c|c}
\hline Parameter & $C$ & $R$ & $P$ & $\sigma$ & $\eta$ & $\theta_{a}$ & $\theta_{+}$ & $\theta_{-}$ \\
\hline Value & 1.5 & 2 & 4 & 0.01 & 2.5 & 26 & 20 & 19 \\
\hline Units & $\mathrm{kWh} /{ }^{\circ} \mathrm{C}$ & ${ }^{\circ} \mathrm{C} / \mathrm{kW}$ & $\mathrm{kW}$ & ${ }^{\circ} \mathrm{C}$ & - & ${ }^{\circ} \mathrm{C}$ & ${ }^{\circ} \mathrm{C}$ & ${ }^{\circ} \mathrm{C}$ \\
\hline
\end{tabular}

Our objective is to exploit the flexibility in the population of TCLs by shaping their power demand within the allowed limits. It is therefore necessary to introduce a control element in the previous model to change the aggregate power consumption. We introduce the rate functions $\mu_{10}(x, t), \quad \mu_{01}(x, t): \mathbb{R} \times \mathbb{R}_{+} \rightarrow \mathbb{R}_{+}$and we modify the way the system naturally switches from (3) to

$$
\begin{array}{r}
\mathbb{P}(m(t+d t)=i \mid m(t)=j, X(t)=x)= \\
=\lambda_{j i}(x) d t+\mu_{j i}(x, t) d t+\mathcal{O}(d t)
\end{array}
$$

with $i \neq j, i, j=0,1$. The implementation of the switching control at the individual TCL level will be discussed in V. Since this approach may introduce a higher switching rate and wear out the devices, an upper bound is introduced

$$
\mu_{01}(x, t) \leq \bar{\mu}_{01}, \quad \mu_{10}(x, t) \leq \bar{\mu}_{10} .
$$

\section{B. Aggregate TCL Model}

Let us denote with $f_{1}(x)$ and $f_{0}(x)$ the drift of (1) in the on and off mode, respectively. Given the initial probability distribution of the TCLs in the two modes as $\rho_{1}(x, 0)$ and $\rho_{0}(x, 0)$, the behavior of a homogeneous population can be described by the probability densities $\rho_{1}(x, t), \rho_{0}(x, t): \mathbb{R} \times$ $\mathbb{R}_{+} \rightarrow \mathbb{R}_{+}$. Their evolution in time is determined by the following system of Fokker Planck equations [12]

$$
\begin{aligned}
\frac{\partial \rho_{i}(x, t)}{\partial t}= & -\frac{\partial}{\partial x}\left(\rho_{i}(x, t) f_{i}(x)\right)+\frac{\sigma^{2}}{2} \frac{\partial^{2} \rho_{i}(x, t)}{\partial x^{2}} \\
& -\left(\lambda_{i j}(x)+\mu_{i j}(x, t)\right) \rho_{i}(x, t) \\
& +\left(\lambda_{j i}(x)+\mu_{j i}(x, t)\right) \rho_{j}(x, t),
\end{aligned}
$$

with $i \neq j, i, j=0,1$.

\section{NumERICAL IMPLEMENTATION AND ANALYSIS OF THE POPUlATION MODEL}

The systems of PDEs (6) do not in general admit a closed form solution. To get more insight into the dynamics, we discretize the PDEs using the Finite Volume Method (FVM) reducing the problem to a system of ODEs. Different discretization techniques could be used, however FVM is chosen due to the mass preserving property [13], i.e. the fact that $\int_{-\infty}^{\infty} \rho_{1}(x, t)+\rho_{0}(x, t) d x=1, \forall t \geq 0$ is guaranteed. We grid the temperature axis uniformly as in Figure 2, where the 
domain is restricted to $J=\left[\theta_{--}, \theta_{++}\right] \supset\left[\theta_{-}, \theta_{+}\right]$since the probability of the temperature being outside $J$ is very small for sufficiently small/large $\theta_{--}$and $\theta_{++}$. Reflecting boundary conditions are introduced at the endpoints of $J$. Let us consider the on mode only, since the exact reasoning

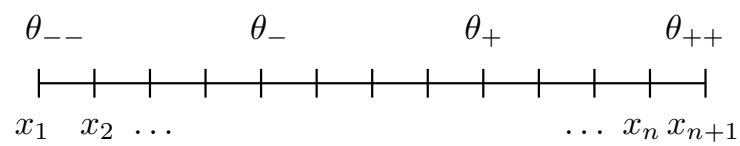

Fig. 2: Temperature axis discretization.

applies also to the off mode. Integrating the first PDE from (6) with $\mu_{01}(x, t)=\mu_{10}(x, t)=0$ in each bin results in

$$
\begin{aligned}
& \frac{d}{d t} \bar{\rho}_{1, k} \Delta x=\left[-\rho_{1}(x, t) f_{1}(x)+\frac{\sigma^{2}}{2} \frac{\partial}{\partial x} \rho_{1}(x, t)\right]_{x_{k}}^{x_{k+1}}+ \\
& -\int_{x_{k}}^{x_{k+1}} \lambda_{10}(x) \rho_{1}(x, t) d x+\int_{x_{k}}^{x_{k+1}} \lambda_{01}(x) \rho_{0}(x, t) d x,
\end{aligned}
$$

where $\bar{\rho}_{1, k}$ is the average of $\rho_{1}(x, t)$ in the $k$-th bin and $\Delta x$ is the length of each bin. By interpolation, $\rho_{1}(x, t)$ can be approximated using the neighboring average values. Finally, by taking the rates to be piecewise constant in each bin, (6) can be transformed into a system of linear differential equations for the column vector $\bar{\rho}=$ $\left[\bar{\rho}_{1,1}, \ldots, \bar{\rho}_{1, n}, \bar{\rho}_{0,1}, \ldots, \bar{\rho}_{0, n}\right]^{\top} \in \mathbb{R}^{2 n}$

$$
\frac{d}{d t} \bar{\rho}(t)=A \bar{\rho}(t) .
$$

The resulting matrix $A \in \mathbb{R}^{2 n \times 2 n}$ can be decomposed into three terms

$$
A=\frac{1}{2 \Delta x}\left[\begin{array}{cc}
A_{0} & 0_{n \times n} \\
0_{n \times n} & A_{1}
\end{array}\right]+A_{2}+A_{3}
$$

with the non-zero elements of $A_{0}$ being

$$
A_{0}(i, j)= \begin{cases}f_{1}\left(x_{i}\right)+\frac{\sigma^{2}}{\Delta x} & j=i-1, \\ f_{1}\left(x_{i}\right)-f_{1}\left(x_{i+1}\right)-\frac{2 \sigma^{2}}{\Delta x} & j=i, \\ -f_{1}\left(x_{i+1}\right)+\frac{\sigma^{2}}{\Delta x} & j=i+1,\end{cases}
$$

for $1<i<n$, and $A_{0}(1,1)=-f_{1}\left(x_{2}\right)-\sigma^{2} / \Delta x$, $A_{0}(1,2)=-f_{1}\left(x_{2}\right)+\sigma^{2} / \Delta x, A_{0}(n, n-1)=f_{1}\left(x_{n}\right)+$ $\sigma^{2} / \Delta x, A_{0}(n, n)=f_{1}\left(x_{n}\right)-\sigma^{2} / \Delta x$. The matrix $A_{1}$ is obtained as $A_{0}$ using $f_{0}(x)$ rather than $f_{1}(x)$. The matrices $A_{2}$ and $A_{3}$ capture the transition rates due to the soft boundaries. Each column of $A_{2}$ and $A_{3}$ contains either all zeros, or only two nonzero elements: a negative one on the diagonal and a positive off diagonal one, with equal magnitude but opposite signs. Since the vector $\bar{\rho}(t)$ represents a probability distribution on the state space, it is required that the discretized system (7) preserves the total mass for all times, that is $\sum_{k=1}^{n}\left(\bar{\rho}_{1, k}(t)+\bar{\rho}_{0, k}(t)\right) \Delta x=1$, and to be positive i.e. $\bar{\rho}_{i, k}(t) \geq 0$ when $\bar{\rho}_{i, k}(0) \geq 0 \forall i, k, \forall t \geq 0$. From (8) and from the structure of $A_{2}, A_{3}$ it follows that sum of the elements in each column of $A$ is zero, thus the total mass is preserved. Positivity can be guaranteed by proving that $A$ is a Metzler matrix. This is true if the space discretization is fine enough, in particular, if

$$
\Delta x<\frac{\sigma^{2}}{\max _{x \in J}\left\{\left|f_{1}(x)\right|,\left|f_{0}(x)\right|\right\}} .
$$

In the following we ensure that this condition is met.

Note that the FVM has transformed the initial system of PDEs into a continuous-time Markov Chain that is irreducible due to the Wiener process that results in the terms $\frac{\sigma^{2}}{\Delta x}$ in (8). These terms enable transition from any state of the Markov chain to any other in finite time. Thus, there exists a unique stationary distribution [14] and for any initial condition, the distribution $\bar{\rho}(t)$ converges to the non trivial solution of $A \bar{\rho}=0$. An example of stationary distribution with parameters from table $\mathrm{I}$ is shown in Figure 3. In all the simulations that follow, we use $\theta_{--}=18.5^{\circ} \mathrm{C}, \theta_{++}=20.5$ ${ }^{\circ} \mathrm{C}$ and $\Delta x=10^{-2}{ }^{\circ} \mathrm{C}, \bar{\mu}_{01}=\bar{\mu}_{10}=2 \cdot 10^{-3}$. These rate limits guarantee that the average time between switches is more than approximately 9 minutes. We choose

$$
\lambda_{01}(x)=\alpha\left(e^{x-\theta_{+}}-1\right)
$$

in $\left[\theta_{+}, \infty\right)$ and zero elsewhere. The same is done symmetrically for $\lambda_{10}(x)$. Unless otherwise stated $\alpha=1$.
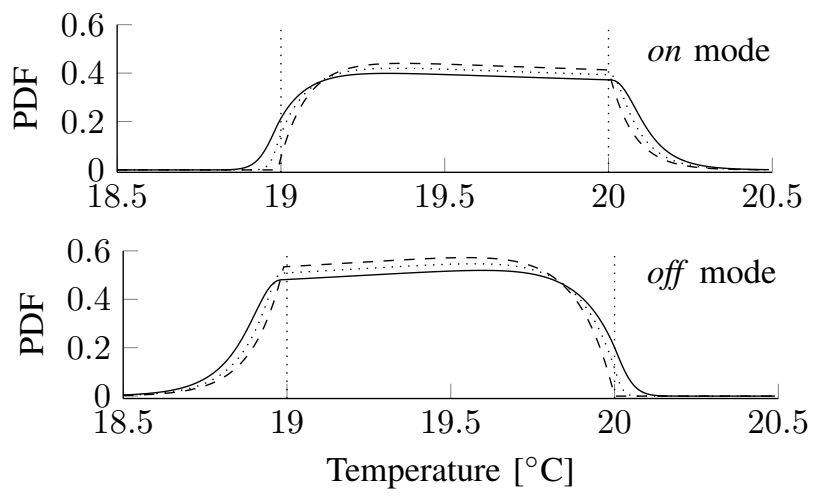

Fig. 3: Stationary distributions with hard boundaries (2) (dashed line), soft boundaries as in (10) with $\alpha=1$ (solid line) and $\alpha=10$ (dotted line).

The aggregate power consumed by the population is proportional to the mass in the on mode $m_{1}(t)$ as

$$
P_{a g g}(t)=P N m_{1}(t)=H \bar{\rho}(t),
$$

where $H:=P N \Delta x\left[1_{1 \times n}, 0_{1 \times n}\right]$ is a row vector. In the following, $\mu_{01}(x, t)$ and $\mu_{10}(x, t)$ are taken to be constant along the temperature axis when inside the deadband $\left[\theta_{-}, \theta_{+}\right]$ with a value of $\mu_{01}(t)$ and $\mu_{10}(t)$, respectively, and zero outside this region. When the control rates $\mu_{01}(t), \mu_{10}(t)$ are introduced, one can still apply the FVM. The result is a bilinear system of the form

$$
\frac{d}{d t} \bar{\rho}(t)=\left(A+\mu_{10}(t) B_{10}+\mu_{01}(t) B_{01}\right) \bar{\rho}(t):=A_{c}(t) \bar{\rho}(t) .
$$

Note that the closed loop matrix $A_{c}(t)$ has the same property as $A$ for all times $t$ : each column sums to zero and $A_{c}(t)$ 
is Metzler, provided (9) is satisfied. Thus, mass is preserved and positivity of $\bar{\rho}(t)$ is ensured.

When the control rates $\mu_{01}(t), \mu_{10}(t)$ are fixed in time, the existence of a unique attracting stationary distribution is guaranteed, since the Markov chain is still finite and irreducible. From a regulating perspective, if the range of stationary power outputs is sufficiently large and the transients are fast, one may consider controlling the system by steering it from one steady state to another. Recall that by (11) the power consumed is proportional to the amount of mass in the on mode $m_{1}(t)$. In Figure 4, $m_{1}(t)$ is computed for different values of the constant control inputs $\mu_{01}$. As shown in

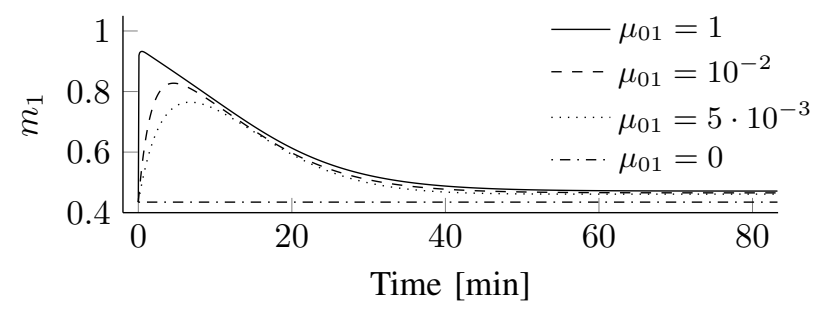

Fig. 4: Evolution of $m_{1}(t)$ for the system (12) with different control inputs starting from the stationary distribution of (7) and increasing $\mu_{01}$ while fixing $\mu_{10}=0$.

Figure 4, the range of power outputs at steady state is much smaller compared to what can be reached in the transients. This can be explained by the following observation. At steady state, the heat extracted from an aggregation of TCLs has to equal the heat inflow. Since the latter is proportional to the average difference in temperature of the system with that of the ambient and the deadband excursion is approximately $1^{\circ} \mathrm{C}$, the possible power values $P_{a g g}$ at steady state are limited. This observation suggests one should harness the flexibility that comes from the transients. In this direction, we turn our attention to the set of desired power trajectories $P_{d}(s): \mathbb{R}_{+} \rightarrow \mathbb{R}$ that the system can track, given a certain initial condition $\bar{\rho}(0)$. Characterizing this set of trajectories is in general very difficult due to the bilinearity in (12). Thus, we provide necessary conditions on feasible power trajectories $P_{d}(s)$ over a horizon $\left[t_{0}, t_{f}\right]$.

\section{Characterizing TCL Population Control POTENTIAL}

In the following the control volume consists of the space within all the buildings where a TCL operates.

Lemma 1: Consider a population of $N$ homogeneous cooling TCLs with hybrid dynamics (1), probabilistic switching (4), rate limits (5) and initial distribution $X(0)$. Then the internal energy of the control volume $e(t)$ is upper and lower bounded at all times $t$ by

$C N\left[\theta_{\bar{\mu}_{01}}-\mathbb{E}(X(0))\right] \leq e(t)-e(0) \leq C N\left[\theta_{\bar{\mu}_{10}}-\mathbb{E}(X(0))\right]$,

where $\theta_{\bar{\mu}_{01}}$ is the mean of the stationary distribution that solves $\left(A+\bar{\mu}_{01} B_{01}\right) \bar{\rho}=0$, and $\theta_{\bar{\mu}_{10}}$ is the mean of the stationary distribution that solves $\left(A+\bar{\mu}_{10} B_{10}\right) \bar{\rho}=0$.
Proof: For a single building the variation in internal energy is proportional to the variation in temperature [15], thus for a finite number of homogeneous units $e(t)-e(0)=$ $\sum_{l} C\left(T_{l}(t)-T_{l}(0)\right)$, where $T_{l}(t)$ represents the temperature of the $l$-th unit. Consequently, for a population we can write

$$
e(t)-e(0)=C N \int_{-\infty}^{+\infty} x \sum_{i=0,1}\left(\rho_{i}(x, t)-\rho_{i}(x, 0)\right) d x .
$$

Thus, the variation in energy is proportional to the variation in the average temperature of the population $e(t)-e(0)=$ $C N[\mathbb{E}(X(t))-\mathbb{E}(X(0))]$. Since the average temperature $\mathbb{E}(X(t))$ increases when mass is moved from the on mode to the off mode, and since the switching rates are constrained by (5), the highest $\mathbb{E}(X(t))$ is the steady state temperature when we apply the constant control $\mu_{10}(t)=\bar{\mu}_{10}$. It follows that the average temperature is naturally upper bounded by $\theta_{\bar{\mu}_{10}}$, obtained a priori as the mean of the stationary distribution that solves $\left(A+\bar{\mu}_{10} B_{10}\right) \bar{\rho}=0$. This results in $e(t)-e(0) \leq C N\left[\theta_{\bar{\mu}_{10}}-\mathbb{E}(X(0))\right]:=\Delta e_{\max }$. With the same reasoning, one finds a lower bound on the expected value of the temperature $\theta_{\bar{\mu}_{01}}$ and shows that $e(t)-e(0) \geq$ $C N\left[\theta_{\bar{\mu}_{01}}-\mathbb{E}(X(0))\right]:=\Delta e_{\text {min }}$.

Given a desired power trajectory $P_{d}(s), s \in[0, t]$, and an initial distribution $X(0)$, we would like to know a priori if (13) is satisfied. The next proposition provides the answer.

Proposition 1: Under the assumption of Lemma 1, consider a desired power trajectory $P_{d}(s), s \in[0, t]$. Denote with $P_{\min }$ and $P_{\max }$ the electric power consumed at steady state when the maximum value of $\mu_{10}$ resp. $\mu_{01}$ is applied.

1) If $-\Delta e_{\max }+\eta P_{\max } t \leq \int_{0}^{t} \eta P_{d}(s) d s \leq-\Delta e_{\min }+$ $\eta P_{\min } t$, then $\Delta e_{\min } \leq e(t)-e(0) \leq \Delta e_{\max }$.

2) If $\int_{0}^{t} \eta P_{d}(s) d s>-\Delta e_{\text {min }}+\eta P_{\text {max }} t$ then $e(t)-$ $e(0)<\Delta e_{\min }$. If $\int_{0}^{t} \eta P_{d}(s) d s<-\Delta e_{\max }+\eta P_{\min } t$ then $e(t)-e(0)>\Delta e_{\max }$.

Proof: Let us first prove 1). From the first principle of thermodynamics [15] we know that

$$
e(t)-e(0)=-\int_{0}^{t} \eta P_{a g g}(s) d s+\int_{0}^{t} I(s) d s,
$$

where $-\eta P_{\text {agg }}(s)$ represents the aggregate heat flow that the ACs extract, while $I(s)$ is the total inflow of heat due to the higher ambient temperature. Note that $I(s) \geq \eta P_{\text {min }}$. This inequality describes the fact that the heat inflow $I(s)$ is always greater than the inflow when the average temperature $\mathbb{E}(X(t))$ is the highest possible. The value of $P_{\min }$ can be easily computed beforehand as $P_{\text {min }}=H \bar{\rho}$, where $\bar{\rho}$ solves $\left(A+\bar{\mu}_{10} B_{10}\right) \bar{\rho}=0$. As a consequence of this estimate and of (15), it follows that

$$
e(t)-e(0) \geq-\int_{0}^{t} \eta P_{a g g}(s) d s+\eta P_{\min } t .
$$

Keeping in mind (13), given a desired power trajectory $P_{d}(s)$, if $\int_{0}^{t} \eta P_{d}(s) d s \leq-\Delta e_{\text {min }}+\eta P_{\text {min }} t$, we can conclude that the constraint $e(t)-e(0) \geq \Delta e_{\min }$ is satisfied. Similarly one can show that if $\int_{0}^{t} \eta P_{d}(s) d s \geq-\Delta e_{\max }+$ 
$\eta P_{\max } t$, then $e(t)-e(0) \leq \Delta e_{\max }$. This proves 1$)$.

To prove 2) note that $I(s) \leq \eta P_{\max }$. This inequality describes the fact that the heat inflow $I(s)$ is always smaller than the inflow when the average temperature $\mathbb{E}(X(t))$ is the lowest possible. The value of $P_{\max }$ can be computed beforehand as $P_{\max }=H \bar{\rho}$, where $\bar{\rho}$ solves $\left(A+\bar{\mu}_{01} B_{01}\right) \bar{\rho}=$ 0 . Thus, one has that

$$
e(t)-e(0) \leq-\int_{0}^{t} \eta P_{a g g}(s) d s+\eta P_{\max } t
$$

If the desired power $P_{d}(s)$ is such that $\int_{0}^{t} \eta P_{d}(s) d s>$ $-\Delta e_{\min }+\eta P_{\text {max }} t$, then from Equation (17), $e(t)-e(0)<$ $\Delta e_{\min }$. Similarly one can prove the second part of 2$)$.

The statement 1) in Proposition 1 guarantees that (13) is satisfied, while the statement 2) certifies the infeasibility of the same inequality, thus providing a necessary condition for $P_{d}(s)$ to be tracked. These conditions on the desired power can be represented graphically, as shown in Figure 5, for the integral of the on mass $m_{1}(t)$, which is proportional to the aggregate power of TCLs.

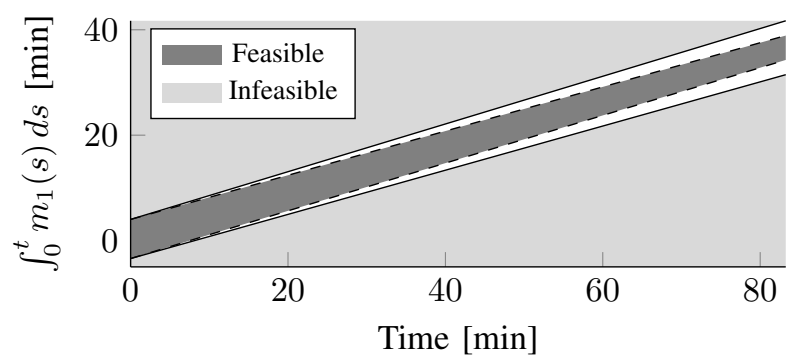

Fig. 5: Feasible and infeasible regions for (13). For trajectories lying in the white portion we cannot a priori guarantee feasibility.

\section{Case Studies}

In this section, we present a feedback controller in order to illustrate the results obtained previously. Furthermore, we provide more insight into energy bounds derived here by discussing the case with heterogeneous parameters and by connecting our work with the previous approaches [10], [9].

\section{A. Control and simulations}

Given a power signal $P_{d}$ provided by a system operator for load following or obtained as a solution of an energy arbitrage problem [10], we use feedback linearization to derive a controller to track it. We take the derivative of the output, which is the aggregate power (11), until the input appears. Using the fact that the dynamics for $\bar{\rho}$ is given by (12), one gets $\dot{P}_{a g g}=H \dot{\bar{\rho}}=H A \bar{\rho}+\mu_{10} H B_{10} \bar{\rho}+\mu_{01} H B_{01} \bar{\rho}$. Thus, the system has relative degree 1 . We then use one of the controls $\mu_{10}$ or $\mu_{01}$ at a single time. If $\dot{P}_{d}-H A \bar{\rho}<0$, then more mass is needed in the off mode and $\mu_{10}$ is used. Since the maximum value of $\mu_{10}$ is upper bounded by (5), we obtain the following controller

$$
\mu_{10}=\min \left\{\frac{\dot{P}_{d}-H A \bar{\rho}}{H B_{10} \bar{\rho}}, \bar{\mu}_{10}\right\} \geq 0 .
$$

Note that the previous equation is meaningful if the denominator of the first term is bounded away from zero, which is always the case when the mass in the deadband of the on mode is non-zero. Similarly $\mu_{01}$ is computed when $\dot{P}_{d}-$ $H A \bar{\rho}>0$. The closed loop system will be an inhomogeneous Markov chain, preserving mass and positivity. The control can be implemented by broadcasting the signals $\mu_{01}(t)$ and $\mu_{10}(t)$ to all the units at discrete instants of time. If for instance, at the discrete time $t_{k}, \mu_{01}\left(t_{k}\right) \neq 0$, each unit in the off mode inside the deadband will draw a random number $r_{k}$ uniformly distributed in the interval $[0,1]$. Recalling (4), if $r_{k} \leq \mu_{01}\left(t_{k}\right) \Delta t$, the unit will switch on.

The control is non-disruptive since we do not change the natural switching outside the deadband. In addition, it is distributed in the sense that given a rate $\mu_{01}$ or $\mu_{10}$ broadcasted by a central aggregator, the TCLs can draw a random number and implement the control. In this formulation, the aggregator requires knowledge of the state $\bar{\rho}$ at each time instant. In general, this information may not be available but can be estimated using aggregate power measurements [3].

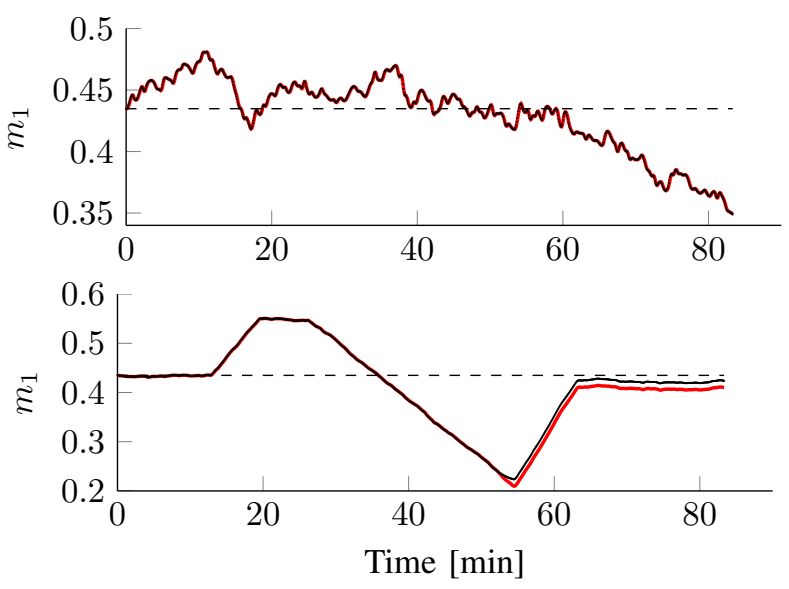

Fig. 6: Simulations of power tracking. The desired and the actual trajectories are in red and black, respectively. The uncontrolled consumption is dashed. The trajectory at the top satisfies the energy constraints and can be delivered, while the one at the bottom does satisfy the constraints but can not be tracked as accurately.

In Figure 6 we present two simulation results using the feedback linearization control. The trajectory in the first plot of Figure 6 satisfies the constraints in (13) and can be tracked. Nevertheless, the conditions previously obtained are only necessary. There could exist a power trajectory that satisfies the derived bounds, but cannot be delivered. This is shown in the second plot of Figure 6.

Difficulty in tracking for trajectories that satisfy the necessary conditions can arise in two cases. First, if the derivative of the desired power trajectory $P_{d}$ is too high, the control saturates due to the rate constraints in (5). Second, when the derivative of $P_{d}$ is kept roughly constant as in the second trajectory of Figure 6, increasingly more mass is taken from the on mode and moved to the off mode, see Figure 7. This decreases the magnitude of the denominator in (18) 
and saturates the controller.

Notice that for any negative or positive derivative of the desired power signal $P_{d}(s)$, there exists a unique $\mu_{10}(s)$ or $\mu_{01}(s)$ that tracks the desired trajectory perfectly. Thus, the only potential way to avoid or postpone saturation is to use state-dependent switching rates $\mu_{01}(x, t), \mu_{10}(x, t)$.

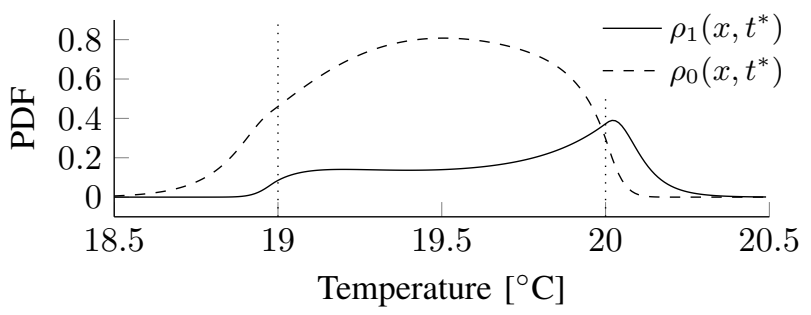

Fig. 7: Distributions for the second trajectory of Figure 6, at time $t^{*}=55$ [min], when tracking is lost.

\section{B. Discussion}

The bounds derived in Proposition 1 are a function of the ambient temperature, size of the TCLs population and their parameters. Furthermore, the energy bounds of Equation (13) for a horizon of $\left[t_{0}, t_{f}\right]$ depend only on the estimate of average temperature at time $t_{0}$, while the bounds on $I(s)$ are independent of the distribution. It is consequently straightforward to compute them.

For heterogeneous parameters one can use a probability density function with an extended state in which the parameters are modeled as additional states. Similar derivation of the continuous-time Markov chain will follow, with the difference that the matrices $A, B_{01}, B_{10}$ will be computed using the updated probability density function evolution. Same reasoning for derivation of bounds can be applied. However, the existence of a unique stationary distribution needs to be shown. Alternatively, the Markov chain model can be identified experimentally for a population of heterogenous TCLs [3]. In this case, we can still use the approach to find the invariant distribution of the Markov chain given the switching rate constraints and thus derive the energy bounds.

The bounds derived in Proposition 1 are closely related to those in [10]. In this work, a first order linear model for the integral of power, referred to as a storage model, is derived. The parameters $\Delta e_{\max }$ and $\Delta e_{\min }$ correspond to the upper and lower bounds on the storage. In addition, the term capturing the natural discharge of the storage model in [10] is equivalent to the inflow term $I$, at steady-state. We derive more accurate upper and lower bounds on the inflow by quantifying the maximum/minimum inflow. The average temperature equivalence with energy used here for derivation of bounds is also connected with the approach in [9]. In this work, by assuming a linear and deterministic model for TCLs, the average temperature dynamics of population is approximated by a linear model. Since such dynamics is an accurate approximation near steady-state operations, the generalized battery model is effective in zero mean fast time scale regulation rather than minutes to hours, as is the case here. Furthermore, with respect to past work, the formulation presented here accounts for practically motivated upper bounds on rate of switching in the derivation of the bounds.

\section{CONCLUSIONS}

We studied the problem of quantifying the set of power trajectories that a population of TCLs can track using probabilistic switching control schemes. Based on the hybrid stochastic model of the individual TCLs, we derived necessary conditions on feasible power trajectories over a horizon of interest. The derivation was based on recognizing the energy of the population as the average temperature, and then using the probability distribution of the population to quantify limits on the average temperature. Future work includes accounting for heterogeneity in the probability density function, estimating the state of the Markov chain using partial measurements and extending the results to TCLs with more complex dynamics and random switching such as electric water heaters.

\section{REFERENCES}

[1] D. Callaway, "Tapping the energy storage potential in electric loads to deliver load following and regulation, with application to wind energy," Energy Conversion and Management, vol. 50, pp. 1389-1400, 2009.

[2] R. Malhamé and C. Chong, "Electric load model synthesis by diffusion approximation of a high-order hybrid-state stochastic system," IEEE Transactions on Automatic Control, vol. 30, no. 9, pp. 854-860, 1985.

[3] J. L. Mathieu, S. Koch, and D. S. Callaway, "State estimation and control of electric loads to manage real-time energy imbalance," Power Systems, IEEE Transactions on, vol. 28, no. 1, pp. 430-440, 2013.

[4] L. C. Totu and R. Wisniewski, "Demand response of thermostatic loads by optimized switching-fraction broadcast," in IFAC World Congress, aug 2014, pp. 9956-9961.

[5] W. Zhang, K. Kalsi, J. Fuller, M. Elizondo, and D. Chassin, "Aggregate model for heterogeneous thermostatically controlled loads with demand response," in Proceedings of IEEE PES General Meeting, San Diego, CA, 2012.

[6] A. C. Kizilkale and R. P. Malhamé, "Mean field based control of power system dispersed energy storage devices for peak load relief," in IEEE Conference on Decision and Control. IEEE, 2013, pp. 4971-4976.

[7] F. Bagagiolo and D. Bauso, "Mean-field games and dynamic demand management in power grids," Dynamic Games and Applications, vol. 4, no. 2, pp. 155-176, 2014.

[8] D. Angeli and P.-A. Kountouriotis, "A stochastic approach to dynamicdemand refrigerator control," Control Systems Technology, IEEE Transactions on, vol. 20, no. 3, pp. 581-592, 2012.

[9] B. M. Sanandaji, H. Hao, K. Poolla, and T. L. Vincent, "Improved battery models of an aggregation of thermostatically controlled loads for frequency regulation," in American Control Conference (ACC), 2014. IEEE, 2014, pp. 38-45.

[10] J. Mathieu, M. Kamgarpour, J. Lygeros, G. Andersson, and D. Callaway, "Arbitraging intraday wholesale energy market prices with aggregations of thermostatic loads," Power Systems, IEEE Transactions on, vol. 30, no. 2, pp. 763-772, March 2015.

[11] A. Abate, M. Prandini, J. Lygeros, and S. Sastry, "Approximation of general stochastic hybrid systems by switching diffusions with random hybrid jumps," in Hybrid Systems: Computation and Control, 11th International Workshop, HSCC 2008, St. Louis, MO, USA, April 2224, 2008. Proceedings, 2008, pp. 598-601.

[12] J. Krystul, A. Bagchi, and H. Blom, "Risk decomposition and assessment methods," Report Hybridge WP8, vol. 1, 2003.

[13] J. H. Ferziger and M. Perić, Computational methods for fluid dynamics. Springer Berlin, 2002, vol. 3.

[14] R. Durrett, Essentials of stochastic processes. Springer Science \& Business Media, 2012.

[15] P. Nag, Engineering Thermodynamics. Tata McGraw Hill, 2005. [Online]. Available: http://books.google.it/books?id=91MZ2ZOb3n8C 